\title{
On the Study of Reverse Degree-Based Topological Properties for the Third Type of $p$ th Chain Hex-Derived Network
}

\author{
Ali N. A. Koam $\mathbb{D}^{1},{ }^{1}$ Ali Ahmad $\mathbb{D}^{2}$ and Ashfaq Ahmed Qummer ${ }^{3}{ }^{3}$ \\ ${ }^{1}$ Department of Mathematics, College of Science, Jazan University, New Campus, Jazan 2097, Saudi Arabia \\ ${ }^{2}$ College of Computer Science \& Information Technology, Jazan University, Jazan, Saudi Arabia \\ ${ }^{3}$ Khawja Freed University of Engineering and Information Technology, Rahim Yar Khan, Pakistan \\ Correspondence should be addressed to Ashfaq Ahmed Qummer; qamarashfaq92@gmail.com
}

Received 30 September 2021; Accepted 19 October 2021; Published 3 November 2021

Academic Editor: Gohar Ali

Copyright (c) $2021 \mathrm{Ali}$ N. A. Koam et al. This is an open access article distributed under the Creative Commons Attribution License, which permits unrestricted use, distribution, and reproduction in any medium, provided the original work is properly cited.

Vertices and edges are made from a network, with the degree of a vertex referring to the number of connected edges. The chance of every vertex possessing a given degree is represented by a network's degree appropriation, which reveals important global network characteristics. Many fields, including sociology, public health, business, medicine, engineering, computer science, and basic sciences, use network theory. Logistical networks, gene regulatory networks, metabolic networks, social networks, and driven networks are some of the most significant networks. In physical, theoretical, and environmental chemistry, a topological index is a numerical value assigned to a molecular structure/network that is used for correlation analysis. Hexagonal networks of dimension $t$ are used to build hex-derived networks, which have a wide range of applications in computer science, medicine, and engineering. For the third type of hex-derived networks, topological indices of reverse degree based are discussed in this study.

\section{Introduction}

A topological descriptor is a numerical value that represents the complete structure of a graph. In the study of topological descriptors, graph theory has shown to be a fruitful field of study. The primary elements of topological indices link the many chemical and physical characteristics of fundamental chemical substances. Vertex-edge-based topological indices are employed in the research of QSAR/QSPR for the prediction of bio-activity of different chemical compounds. With the dimension $p$, hexagonal networks create hex-derived networks, which have a wide range of implementations in engineering, computer science, and also medicine. In [1], researchers created a new form of graph known as a "third type of hex-derived networks" [2,3] and continued this work by calculating degree-based topological descriptors for these networks, in which they computed exact values of some vertex-edge named topological indices for this network.

Researchers have used graph theory to develop a range of helpful tools, including graph labeling, topological indices, and finding numbers. The subject of graph theory has several applications and implementations in various fields of study, including chemistry, medicine, and engineering. A polynomial, a series of integers, a numeric value, or a matrix can all be used to identify a graph. A chemical compound can be represented as a graph (or a diagram) or usually denoted as a molecular graph, nodes played a role of atoms, and the bonding between atoms is usually labeled as edges in the molecular graph theory. Recently, a new topic called cheminformatics was established, which is a mix of chemistry, information science, and mathematics, in which the QSPR/QSAR connection, bio-activity, and characterization of chemical compounds are investigated and reported in [4].

The topological descriptor is a numerical number associated with chemical compositions that maintain the relationship between chemical structures and a variety of physico-chemical characteristics, biological activity, and chemical reactivity. To describe the topology of a chemical network, it translated into a number, which is further used to create topological indices. Distance-based topological indices, degree-based topological indices, and counting-related topological indices are some of the most common 
forms of topological indices for graphs. Many academics have recently discovered topological indices for studying basic features of molecular graphs or networks. In [5-12], these networks have extremely compelling topological qualities that have been examined in distinct characteristics.

Let $p$ and $q$ represent the number of rows and number of triangles in each row of third-type $p$ th chain hex-derived networks $G_{p, q}^{3}$, respectively, shown in Figure 1 . Let $G$ be a simple connected network, with a set of vertex and edges denoted by $V$ and $E$, respectively. $|V|$ represents the order of $G$ and $|E|$ represents the size of $G$. Let $d_{\theta}$ be the degree of a vertex $\theta \in V$ in $G$ and $\mathfrak{R}_{\theta}$ be its reverse degree that was introduced by Kulli [13] and defined as $\mathfrak{R}_{\theta}=1+\Delta-d_{\theta}$, where $\Delta$ denoted the maximum degree of the given graph. Let $\mathbf{E}_{\mathfrak{R}_{\theta}, \mathfrak{R}_{9}}$ represent the edge partition of the given graph based on reverse degree of end vertices of an edge $\theta \vartheta \in E$ and $\left|\mathbf{E}_{\mathfrak{R}_{\theta}, \Re_{9}}\right|$ represent its cardinality.

We define general reverse degree topological invariant

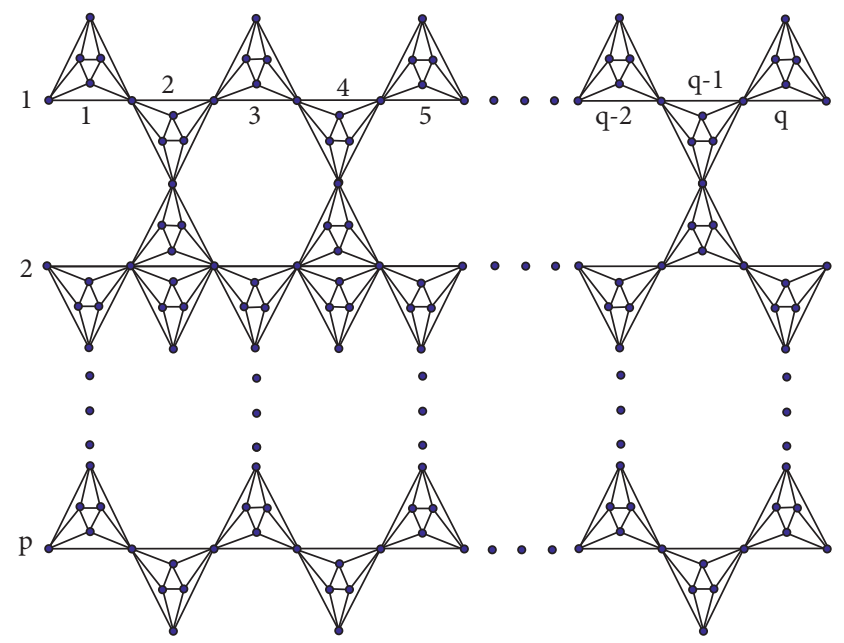

FIgURE 1: Third-type chain hex-derived network $G_{p, q}^{3}$. $\mathbf{T}(G)$ as follows:

$$
\mathscr{T}(G)=\sum_{\theta \vartheta \in E(G)} \lambda\left(\mathfrak{R}_{\theta}, \mathfrak{R}_{\vartheta}\right)
$$

If $\lambda\left(\mathfrak{R}_{\theta}, \mathfrak{R}_{\vartheta}\right)=\left(\mathfrak{R}_{\theta} \times \mathfrak{R}_{\vartheta}\right)^{\alpha}$, then $\mathscr{T}(G)$ represents the general reverse Randić index $\left(\mathfrak{R} \mathbb{R}_{\alpha}(G)\right)$ for $\alpha=\frac{1}{2},-\frac{1}{2}, 1,-1$, If $\alpha=1$, then it is known as the second reverse Zagreb index $\left(\Re \mathbb{M}_{2}(G)\right)$,

If $\lambda\left(\mathfrak{R}_{\theta}, \mathfrak{R}_{\vartheta}\right)=\sqrt{\frac{\mathfrak{R}_{\theta}+\mathfrak{R}_{\vartheta}-2}{\mathfrak{R}_{\theta} \times \mathfrak{R}_{\vartheta}}}$ then $\mathscr{T}(G)$ represents the reverse atom - bond connectivity index $(\mathfrak{R A} \mathbb{B C}(G))$,

If $\lambda\left(\mathfrak{R}_{\theta}, \mathfrak{R}_{\vartheta}\right)=\frac{2 \sqrt{\mathfrak{R}_{\theta} \times \mathfrak{R}_{\vartheta}}}{\mathfrak{R}_{\theta}+\mathfrak{R}_{\vartheta}}$, then $\mathscr{T}(G)$ represents the reverse geometric - arithmetic index $(\mathfrak{R G A}(G))$,

If $\lambda\left(\mathfrak{R}_{\theta}, \mathfrak{R}_{\vartheta}\right)=\left(\mathfrak{R}_{\theta}+\mathfrak{R}_{\vartheta}\right)$, then $\mathscr{T}(G)$ represents the first reverse Zagreb index $\left(\mathfrak{R M}_{1}(G)\right)$,

If $\lambda\left(\mathfrak{R}_{\theta}, \mathfrak{R}_{\vartheta}\right)=\left(\mathfrak{R}_{\theta}+\mathfrak{R}_{\vartheta}\right)^{2}$, then $\mathscr{T}(G)$ represents the reverse hyper Zagreb index $(\mathfrak{R} \nVdash \mathrm{M}(G))$,

If $\lambda\left(\mathfrak{R}_{\theta}, \mathfrak{R}_{\vartheta}\right)=\left(\left(\mathfrak{R}_{\theta}\right)^{2}+\left(\mathfrak{R}_{\vartheta}\right)^{2}\right)$, then $\mathscr{T}(G)$ represents the reverse forgotten index $(\mathfrak{R} \mathbb{F}(G))$,

If $\lambda\left(\mathfrak{R}_{\theta}, \mathfrak{R}_{\vartheta}\right)=\left(\mathfrak{R}_{\theta}+\mathfrak{R}_{\vartheta}\right)^{\alpha}\left(\mathfrak{R}_{\theta} \times \mathfrak{R}_{\vartheta}\right)^{\beta}$, then $\mathscr{T}(G)$ represents the first reverse redefined index $\left(\Re \mathbb{R} \mathbb{Z}_{1}(G)\right)$ for $\alpha=1, \beta=-1$, the second reverse redefined index $\left(\Re \mathbb{R} \mathbb{Z}_{2}(G)\right)$ for $\alpha=-1, \beta=1$, the third reverse redefined index $\left(\Re \mathbb{R} \mathbb{Z}_{3}(G)\right)$ for $\alpha=1, \beta=1$.

For latest results on topological descriptors for different chemical and computer networks and for general graphs, we refer to see [14-25]. In this current research work, we determine the exact values of all the above reverse indices. 


\section{Structure of Third-Type Hex- Derived Networks}

With the help of complete graphs of order $3\left(K_{3}\right)$, Chen et al. [26] assembled a hexagonal mesh. In terms of chemistry, these $K_{3}$ graphs are also called oxide graphs. Figure 1 is obtained by joining these $K_{3}$ graphs. Two-dimensional mesh graph HX(2) (see Figure 2(a)) is obtained by joining six $K_{3}$ graphs and three-dimensional mesh graph $\mathrm{HX}(3)$ (see Figure 2(b)) is obtained by putting $K_{3}$ graphs around all sides of $\mathrm{HX}(2)$ [27]. Furthermore, repeating the same process by putting the $t K_{3}$ graph around each hexagon, we obtained the $t^{\text {th }}$ hexagonal mesh. We have to note that the one-dimensional hexagonal mesh graph does not exist.

The novel network, labeled the third category of hexderived networks, was developed in [1]. In [2, 3], they defined the graphically construction algorithm for the third type of hexagonal hex-derived network $\operatorname{HHDN} 3(t)$. Huo el at. [28] explained the graphical construction algorithm for $m^{\text {th }}$ chain hex-derived network of third type. In this paper, we denote it by $G_{p, q}^{3}$, and different priorities of $p$ and $q$ the chain hex-derived networks are shown in Figure 3. In [29-33], you may find related research that utilizes this idea and that may benefit from the new research's visions.

\section{Main Results}

In this section, we study the third-type $p$ th chain hex-derived networks $G_{p, q}^{3}$ in the following three cases.

(i) Case 1: for $p=q,(p, q) \geq 1$.

(ii) Case 2: for $p<q, p$ is odd and $q$ is a natural number. For $p>q, p$ is odd and $q$ is a natural number. For $p<q, p$ and $q$ both are even. For $p>q, p$ and $q$ both are even.

(iii) Case 3: for $p<q, p$ is even and $q$ is odd. For $p>q, p$ is even and $q$ is odd.

3.1. Results for Case 1. We provide a formula that would be used to calculate any reverse degree topological descriptors of Case 1 for $G_{p, q}^{3}$.

Lemma 1. Let $G_{p, q}^{3}$ be a third-type $p$ th chain hex-derived networks. Then,

$$
\begin{aligned}
\mathscr{T}_{1}\left(G_{p, q}^{3}\right)= & 3 p q(\lambda(1,1)+2 \lambda(1,5)+\lambda(5,5)) \\
& +3(p+q)(\lambda(5,5)-\lambda(1,1))+2(\lambda(1,1) \\
& -2 \lambda(1,5)+\lambda(5,5)) .
\end{aligned}
$$

Proof. The graph $G_{p, q}^{3}$ contains $12 p q$ edges and maximum degree in $G_{p, q}^{3}$ graph is 8 . There are two types of reverse degree vertices in $G_{p, q}^{3}$ that are 1 and 5 . Let us partition the edges of $G_{p, q}^{3}$ according to its reverse degrees according to Case 1 as

$$
\begin{aligned}
& \mathbf{E}_{1,1}=\left\{\theta \vartheta \in E\left(G_{p, q}^{3}\right): \mathfrak{R}_{\theta}=1, \mathfrak{R}_{\vartheta}=1\right\}, \\
& \mathbf{E}_{1,5}=\left\{\theta \vartheta \in E\left(G_{p, q}^{3}\right): \mathfrak{R}_{\theta}=1, \mathfrak{R}_{\vartheta}=5\right\}, \\
& \mathbf{E}_{5,5}=\left\{\theta \vartheta \in E\left(G_{p, q}^{3}\right): \mathfrak{R}_{\theta}=5, \mathfrak{R}_{\vartheta}=5\right\} .
\end{aligned}
$$

Note that $E\left(G_{p, q}^{3}\right)=\mathbf{E}_{1,1} \cup \mathbf{E}_{1,5} \cup \mathbf{E}_{5,5} \quad$ and $\left|\mathbf{E}_{1,1}\right|=3 p q-3 p-3 q+2, \quad\left|\mathbf{E}_{1,5}\right|=6 p q-4, \quad$ and $\left|\mathbf{E}_{5,5}\right|=3 p q+3 p+3 q+2$. Hence,

$$
\begin{aligned}
\mathscr{T}_{1}\left(G_{p, q}^{3}\right)= & \sum_{\theta \vartheta \in E} \lambda\left(G_{p, q}^{3}\right) \\
= & \sum_{\theta \vartheta \in \mathbf{E}_{1,1}} \lambda(1,1)+\sum_{\theta \vartheta \in \mathbf{E}_{1,5}} \lambda(1,5)+\sum_{\theta \vartheta \in \mathbf{E}_{5,5}} \lambda(5,5) \\
= & (3 p q-3 p-3 q+2) \lambda(1,1)+(6 p q-4) \lambda(1,5) \\
& +(3 p q+3 p+3 q+2) \lambda(5,5) .
\end{aligned}
$$

After simplification, we obtain

$$
\begin{aligned}
\mathscr{T}_{1}\left(G_{p, q}^{3}\right)= & 3 p q(\lambda(1,1)+2 \lambda(1,5)+\lambda(5,5)) \\
& +3(p+q)(\lambda(5,5)-\lambda(1,1))+2(\lambda(1,1) \\
& -2 \lambda(1,5)+\lambda(5,5)) .
\end{aligned}
$$

Theorem 1. The general reverse Randić index of $G_{p, q}^{3}$ is equal to

$$
\Re \mathbb{R}_{\alpha}\left(G_{p, q}^{3}\right)= \begin{cases}108 p q+72(p+q)+32, & \text { for } \alpha=1, \\ (18+6 \sqrt{5}) p q+12(p+q)+12-4 \sqrt{5}, & \text { for } \alpha=\frac{1}{2}, \\ \left(\frac{18}{5}+\frac{6 \sqrt{5}}{5}\right) p q-\frac{12}{5}(p+q)+\frac{12}{5}-\frac{4 \sqrt{5}}{5}, & \text { for } \alpha=\frac{-1}{2}, \\ \frac{108}{25} p q-\frac{72}{25}(p+q)+\frac{32}{25}, & \text { for } \alpha=-1 .\end{cases}
$$



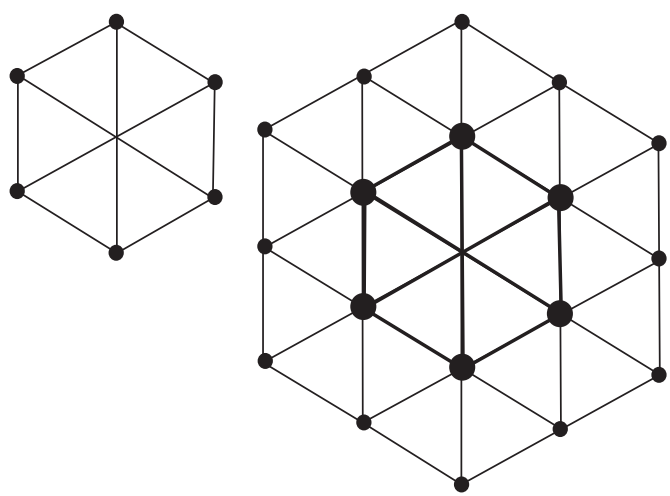

(a)

(b)

Figure 2: Hexagonal meshes: (a) HX (2) and (b) HX (3).

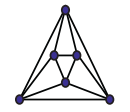

(a)

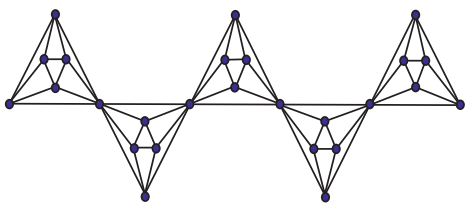

(b)

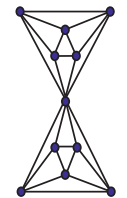

(c)

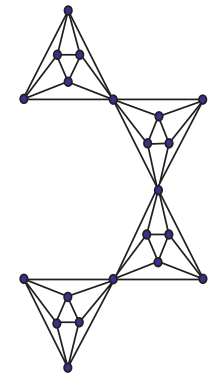

(d)

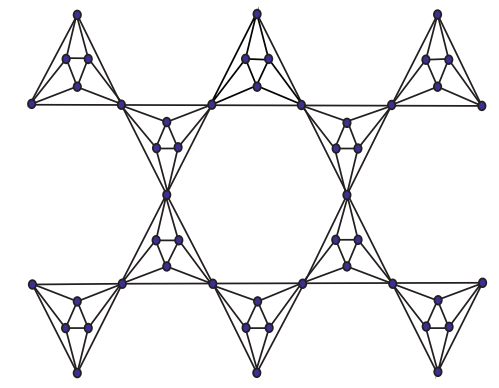

(e)

FIgURE 3: Chain hex-derived networks $G_{p, q}^{3}$ for different priorities of $p$ and $q$.

Proof. For $\mathfrak{R} \mathbb{R}_{\alpha}\left(G_{p, q}^{3}\right)$ which is the general reverse Randić index of $G_{p, q}^{3}, \quad$ from equation (2), we have $\lambda\left(\mathfrak{R}_{\theta}, \mathfrak{R}_{\vartheta}\right)=\left(\mathfrak{R}_{\theta} \times \mathfrak{R}_{\vartheta}\right)^{\alpha} ; \quad$ therefore, $\quad \lambda(1,1)=1$, $\lambda(1,5)=(5)^{\alpha}$, and $\lambda(5,5)=(25)^{\alpha}$. Thus, by Lemma 1 ,

$$
\begin{aligned}
\left.\mathfrak{R} \mathbb{R}_{\alpha}\left(G_{p, q}^{3}\right)\right)= & 3 p q\left(1+2(5)^{\alpha}+(25)^{\alpha}\right) \\
& +3(p+q)\left((25)^{\alpha}-1\right)+2\left(1-2(5)^{\alpha}+(25)^{\alpha}\right) .
\end{aligned}
$$

Put $\alpha=1$, and we have

$$
\left.\Re \mathbb{R}_{1}\left(G_{p, q}^{3}\right)\right)=108 p q+72(p+q)+32 .
$$

Put $\alpha=(1 / 2)$, and we have

$$
\left.\mathfrak{R} \mathbb{R}_{(1 / 2)}\left(G_{p, q}^{3}\right)\right)=(18+6 \sqrt{5}) p q+12(p+q)+12-4 \sqrt{5} .
$$

Put $\alpha=(-1 / 2)$, and we have

$\left.\mathfrak{R} \mathbb{R}_{(-1 / 2)}\left(G_{p, q}^{3}\right)\right)=\left(\frac{18}{5}+\frac{6 \sqrt{5}}{5}\right) p q-\frac{12}{5}(p+q)+\frac{12}{5}-\frac{4 \sqrt{5}}{5}$.

Put $\alpha=-1$, and we have

$$
\left.\Re \mathbb{R}_{-1}\left(G_{p, q}^{3}\right)\right)=\frac{108}{25} p q-\frac{72}{25}(p+q)+\frac{32}{25} .
$$

Theorem 2. Let $G_{p, q}^{3}$ be a third-type $p$ th chain hex-derived networks. Then, the reverse atom-bond connectivity index is $\mathfrak{R A B C}\left(G_{p, q}^{3}\right)=\left(\frac{12 \sqrt{5}}{5}+\frac{6 \sqrt{2}}{5}\right) p q-\frac{8 \sqrt{5}}{5}+\frac{2(3 p+3 q+2) \sqrt{2}}{5}$.

The reverse geometric-arithmetic index is

$$
\Re \mathrm{GA}\left(G_{p, q}^{3}\right)=(6+2 \sqrt{5}) p q+4-\frac{4 \sqrt{5}}{3} .
$$

The first reverse Zagreb index is

$$
\left.\mathfrak{R M} \mathbb{M}_{1}\left(G_{p, q}^{3}\right)\right)=72 p q+24 p+24 q .
$$

The reverse hyper-Zagreb index is

$$
\mathfrak{R} \llbracket \mathrm{M}\left(G_{p, q}^{3}\right)=528 p q+288 p+288 q+64 .
$$

The reverse forgotten index is

$$
\mathfrak{R} \mathbb{F}\left(G_{p, q}^{3}\right)=312 p q+144 p+144 q .
$$

Proof. For $\mathfrak{R A B C}\left(G_{p, q}^{3}\right)$ which is the reverse atom-bond connectivity index of $G_{p, q}^{3}$, from equation (3), we have $\lambda\left(\mathfrak{R}_{\theta}, \mathfrak{R}_{\vartheta}\right)=\sqrt{\left(\mathfrak{R}_{\theta}+\mathfrak{R}_{\vartheta}-2\right) / \mathfrak{R}_{\theta} \times \mathfrak{R}_{\vartheta}} ;$ therefore, $\lambda(1,1)$ 
$=0, \lambda(1,5)=\sqrt{4 / 5}$, and $\lambda(5,5)=(\sqrt{8} / 5)$. Thus, by Lemma 1 and after simplification,

$\mathfrak{R A B C}\left(G_{p, q}^{3}\right)=\left(\frac{12 \sqrt{5}}{5}+\frac{6 \sqrt{2}}{5}\right) p q-\frac{8 \sqrt{5}}{5}+\frac{2(3 p+3 q+2) \sqrt{2}}{5}$.

For $\mathfrak{R G A}\left(G_{p, q}^{3}\right)$ which is the reverse geometric-arithmetic index of $G_{p, q}^{3}$, from equation (4), we have $\lambda\left(\mathfrak{R}_{\theta}, \mathfrak{R}_{\vartheta}\right)=\left(2 \sqrt{\mathfrak{R}_{\theta} \times \mathfrak{R}_{\vartheta}} /\left(\mathfrak{R}_{\theta}+\mathfrak{R}_{\vartheta}\right)\right)$; therefore, $\lambda(1,1)$ $=1, \lambda(1,5)=(\sqrt{5} / 3)$, and $\lambda(5,5)=1$. Thus, by Lemma 1 and after simplification,

$$
\Re \mathrm{GA}\left(G_{p, q}^{3}\right)=(6+2 \sqrt{5}) p q+4-\frac{4 \sqrt{5}}{3} .
$$

For $\Re \mathbb{M}_{1}\left(G_{p, q}^{3}\right)$ which is the first reverse Zagreb index of $G_{p, q}^{3}$, from equation (5), we have $\lambda\left(\mathfrak{R}_{\theta}, \mathfrak{R}_{\vartheta}\right)=\left(\mathfrak{R}_{\theta}+\mathfrak{R}_{\vartheta}\right)$; therefore, $\lambda(1,1)=2, \lambda(1,5)=6$, and $\lambda(5,5)=10$. Thus, by Lemma 1 and after simplification,

$$
\left.\mathfrak{R} \mathbb{M}_{1}\left(G_{p, q}^{3}\right)\right)=72 p q+24 p+24 q .
$$

For $\mathfrak{R} \llbracket M\left(G_{p, q}^{3}\right)$ which is the first reverse hyper-Zagreb index of $G_{p, q}^{3}$, from equation (6), we have $\lambda\left(\mathfrak{R}_{\theta}, \mathfrak{R}_{\vartheta}\right)=\left(\mathfrak{R}_{\theta}+\mathfrak{R}_{\vartheta}\right)^{2} ; \quad$ therefore, $\quad \lambda(1,1)=4$, $\lambda(1,5)=36$, and $\lambda(5,5)=100$. Thus, by Lemma 1 and after simplification,

$$
\mathfrak{R} \Vdash \mathrm{M}\left(G_{p, q}^{3}\right)=528 p q+288 p+288 q+64 .
$$

For $\mathfrak{R} \mathbb{F}\left(G_{p, q}^{3}\right)$ which is the reverse forgotten index of $G_{p, q}^{3}$, from equation (7), we have $\lambda\left(\mathfrak{R}_{\theta}, \mathfrak{R}_{\vartheta}\right)=\left(\left(\mathfrak{R}_{\theta}\right)^{2}+\left(\mathfrak{R}_{\vartheta}\right)^{2}\right) ; \quad$ therefore, $\quad \lambda(1,1)=2$, $\lambda(1,5)=26$, and $\lambda(5,5)=50$. Thus, by Lemma 1 and after simplification,

$$
\mathfrak{R} \mathbb{F}\left(G_{p, q}^{3}\right)=312 p q+144 p+144 q .
$$

Theorem 3. Let $G_{p, q}^{3}$ be a third-type $p$ th chain hex-derived networks. Then, the first reverse redefined index is

$$
\mathfrak{R} \mathbb{R} \mathbb{Z}_{1}\left(G_{p, q}^{3}\right)=\frac{72 p q}{5}-\frac{24 p}{5}-\frac{24 q}{5} .
$$

The second reverse redefined index is

$$
\mathfrak{R} \mathbb{R} \mathbb{Z}_{2}\left(G_{p, q}^{3}\right)=14 p q+6 p+6 q+\frac{8}{3} .
$$

The third reverse redefined index is

$$
\mathfrak{R} \mathbb{R} \mathbb{Z}_{3}\left(G_{p, q}^{3}\right)=936 p q+744 p+744 q+384 .
$$

Proof. For $\mathfrak{R} \mathbb{R} \mathbb{Z}_{1}\left(G_{p, q}^{3}\right)$ which is the first reverse redefined index of $G_{p, q}^{3}$, from equation (8), we have $\lambda\left(\mathfrak{R}_{\theta}, \mathfrak{R}_{\vartheta}\right)=\left(\mathfrak{R}_{\theta}+\mathfrak{R}_{\vartheta}\right)^{1}\left(\mathfrak{R}_{\theta} \times \mathfrak{R}_{\vartheta}\right)^{-1} ; \quad$ therefore, $\lambda(1,1)=2, \lambda(1,5)=(6 / 5)$, and $\lambda(5,5)=(2 / 5)$. Thus, by Lemma 1 and after simplification,

$$
\mathfrak{R} \mathbb{R} \mathbb{Z}_{1}\left(G_{p, q}^{3}\right)=\frac{72 p q}{5}-\frac{24 p}{5}-\frac{24 q}{5} .
$$

For $\Re \mathbb{R} \mathbb{Z}_{2}\left(G_{p, q}^{3}\right)$ which is the second reverse redefined index of $G_{p, q}^{3}$, from equation (8), we have $\lambda\left(\mathfrak{R}_{\theta}, \mathfrak{R}_{\vartheta}\right)=\left(\mathfrak{R}_{\theta}+\mathfrak{R}_{\vartheta}\right)^{-1}\left(\mathfrak{R}_{\theta} \times \mathfrak{R}_{\vartheta}\right)^{1} ; \quad$ therefore, $\lambda(1,1)=(1 / 2), \lambda(1,5)=(5 / 6)$, and $\lambda(5,5)=(5 / 2)$. Thus, by Lemma 1 and after simplification,

$$
\Re \mathbb{R} \mathbb{Z}_{2}\left(G_{p, q}^{3}\right)=14 p q+6 p+6 q+\frac{8}{3} .
$$

For $\mathfrak{R} \mathbb{R} \mathbb{Z}_{3}\left(G_{p, q}^{3}\right)$ which is the third reverse redefined index of $G_{p, q}^{3}$, from equation (8), we have $\lambda\left(\mathfrak{R}_{\theta}, \mathfrak{R}_{\vartheta}\right)=\left(\mathfrak{R}_{\theta}+\mathfrak{R}_{\vartheta}\right)\left(\mathfrak{R}_{\theta} \times \mathfrak{R}_{\vartheta}\right)$; therefore, $\lambda(1,1)=2$, $\lambda(1,5)=30$, and $\lambda(5,5)=250$. Thus, by Lemma 1 and after simplification,

$$
\Re \mathbb{R} \mathbb{Z}_{3}\left(G_{p, q}^{3}\right)=936 p q+744 p+744 q+384 .
$$

3.2. Results for Case 2. We provide a formula that would be used to calculate any reverse degree topological descriptors of Case 2 for $G_{p, q}^{3}$.

Lemma 2. Let $G_{p, q}^{3}$ be a third-type $p$ th chain hex-derived networks. Then,

$$
\begin{aligned}
\mathscr{T}_{2}\left(G_{p, q}^{3}\right)= & 3 p q(\lambda(1,1)+2 \lambda(1,5)+\lambda(5,5)) \\
& +2(2 p+q)(\lambda(5,5)-\lambda(1,1))+2(\lambda(1,1) \\
& -2 \lambda(1,5)+\lambda(5,5)) .
\end{aligned}
$$

Proof. The graph $G_{p, q}^{3}$ contains $12 p q$ edges and maximum degree in $G_{p, q}^{3}$ graph is 8 . There are two types of reverse degree vertices in $G_{p, q}^{3}$ that are 1 and 5. Let us partition the edges of $G_{p, q}^{3}$ according to its reverse degrees according to Case 2 as

$$
\begin{aligned}
& \mathbf{E}_{1,1}=\left\{\theta \vartheta \in E\left(G_{p, q}^{3}\right): \mathfrak{R}_{\theta}=1, \mathfrak{R}_{\vartheta}=1\right\}, \\
& \mathbf{E}_{1,5}=\left\{\theta \vartheta \in E\left(G_{p, q}^{3}\right): \mathfrak{R}_{\theta}=1, \mathfrak{R}_{\vartheta}=5\right\}, \\
& \mathbf{E}_{5,5}=\left\{\theta \vartheta \in E\left(G_{p, q}^{3}\right): \mathfrak{R}_{\theta}=5, \mathfrak{R}_{\vartheta}=5\right\} .
\end{aligned}
$$

Note that $E\left(G_{p, q}^{3}\right)=\mathbf{E}_{1,1} \cup \mathbf{E}_{1,5} \cup \mathbf{E}_{5,5} \quad$ and $\left|\mathbf{E}_{1,1}\right|=3 p q-4 p-2 q+2, \quad\left|\mathbf{E}_{1,5}\right|=6 p q-4, \quad$ and $\left|\mathbf{E}_{5,5}\right|=3 p q+4 p+2 q+2$. Hence,

$$
\begin{aligned}
\mathscr{T}_{2}\left(G_{p, q}^{3}\right)= & \sum_{\theta \vartheta \in E} \lambda\left(G_{p, q}^{3}\right) \\
= & \sum_{\theta \vartheta \in \mathrm{E}_{1,1}} \lambda(1,1)+\sum_{\theta \vartheta \in \mathrm{E}_{1,5}} \lambda(1,5)+\sum_{\theta \vartheta \in \mathrm{E}_{5,5}} \lambda(5,5) \\
= & (3 p q-4 p-2 q+2) \lambda(1,1)+(6 p q-4) \lambda(1,5) \\
& +(3 p q+4 p+2 q+2) \lambda(5,5) .
\end{aligned}
$$

After simplification, we obtain 


$$
\begin{aligned}
\mathscr{T}_{2}\left(G_{p, q}^{3}\right)= & 3 p q(\lambda(1,1)+2 \lambda(1,5)+\lambda(5,5)) \\
& +2(2 p+q)(\lambda(5,5)-\lambda(1,1))+2(\lambda(1,1) \\
& -2 \lambda(1,5)+\lambda(5,5)) .
\end{aligned}
$$

Theorem 4. The general reverse Randić index of $G_{p, q}^{3}$ is equal (38) to

$$
\mathfrak{R} \mathbb{R}_{\alpha}\left(G_{p, q}^{3}\right)= \begin{cases}108 p q+96 p+48 q+32, & \text { for } \alpha=1 \\ (18+6 \sqrt{5}) p q+16 p+8 q+12-4 \sqrt{5}, & \text { for } \alpha=\frac{1}{2} \\ \left(\frac{18}{5}+\frac{6 \sqrt{5}}{5}\right) p q-\frac{16 p}{5}-\frac{8}{5} q+\frac{12}{5}-\frac{4 \sqrt{5}}{5}, & \text { for } \alpha=\frac{-1}{2} \\ \frac{108}{25} p q-\frac{96}{25} p-\frac{48}{25} q+\frac{32}{25}, & \text { for } \alpha=-1\end{cases}
$$

Proof. For $\mathfrak{R} \mathbb{R}_{\alpha}\left(G_{p, q}^{3}\right)$ which is the general reverse Randić index of $G_{p, q}^{3}$, from equation (2), we have $\lambda\left(\mathfrak{R}_{\theta}, \mathfrak{R}_{\vartheta}\right)=\left(\mathfrak{R}_{\theta} \times \mathfrak{R}_{\vartheta}\right)^{\alpha} ; \quad$ therefore, $\quad \lambda(1,1)=1$, $\lambda(1,5)=(5)^{\alpha}$, and $\lambda(5,5)=(25)^{\alpha}$. Thus, by Lemma 2 ,

$$
\begin{aligned}
\left.\Re \mathbb{R}_{\alpha}\left(G_{p, q}^{3}\right)\right)= & 3 p q\left(1+2(5)^{\alpha}+(25)^{\alpha}\right) \\
& +2(2 p+q)\left((25)^{\alpha}-1\right)+2\left(1-2(5)^{\alpha}+(25)^{\alpha}\right) .
\end{aligned}
$$

Put $\alpha=1$, and we have

$$
\left.\mathfrak{R} \mathbb{R}_{1}\left(G_{p, q}^{3}\right)\right)=108 p q+96 p+48 q+32 .
$$

Put $\alpha=(1 / 2)$, and we have

$$
\left.\mathfrak{R} \mathbb{R}_{(1 / 2)}\left(G_{p, q}^{3}\right)\right)=(18+6 \sqrt{5}) p q+16 p+8 q+12-4 \sqrt{5} .
$$

Put $\alpha=(-1 / 2)$, and we have

$$
\left.\Re \mathbb{R}_{(-1 / 2)}\left(G_{p, q}^{3}\right)\right)=\left(\frac{18}{5}+\frac{6 \sqrt{5}}{5}\right) p q-\frac{16 p}{5}-\frac{8}{5} q+\frac{12}{5}-\frac{4 \sqrt{5}}{5} \text {. }
$$

Put $\alpha=-1$, and we have

$$
\left.\Re \mathbb{R}_{-1}\left(G_{p, q}^{3}\right)\right)=\frac{108}{25} p q-\frac{96}{25} p-\frac{48}{25} q+\frac{32}{25} .
$$

Theorem 5. Let $G_{p, q}^{3}$ be a third-type $p$ th chain hex-derived networks. Then, the reverse atom-bond connectivity index is

$$
\mathfrak{R A B C}\left(G_{p, q}^{3}\right)=\left(\frac{12 \sqrt{5}}{5}+\frac{6 \sqrt{2}}{5}\right) p q-\frac{8 \sqrt{5}}{5}+\frac{2(4 p+2 q+2) \sqrt{2}}{5} .
$$

The reverse geometric-arithmetic index is

$$
\mathfrak{R G A}\left(G_{p, q}^{3}\right)=(6+2 \sqrt{5}) p q+4-\frac{4 \sqrt{5}}{3} .
$$

The first reverse Zagreb index is

$$
\left.\mathfrak{R M} \mathbb{M}_{1}\left(G_{p, q}^{3}\right)\right)=72 p q+32 p+16 q
$$

The reverse hyper-Zagreb index is

$$
\mathfrak{R} \boxplus M\left(G_{p, q}^{3}\right)=528 p q+384 p+192 q+64 .
$$

The reverse forgotten index is

$$
\mathfrak{R} \mathbb{F}\left(G_{p, q}^{3}\right)=312 p q+192 p+96 q .
$$

Proof. For $\mathfrak{R A B C}\left(G_{p, q}^{3}\right)$ which is the reverse atom-bond connectivity index of $G_{p, q}^{3}$, from equation (3), we have $\lambda\left(\mathfrak{R}_{\theta}, \mathfrak{R}_{\vartheta}\right)=\sqrt{\left(\mathfrak{R}_{\theta}+\mathfrak{R}_{\vartheta}-2\right) / \mathfrak{R}_{\theta} \times \mathfrak{R}_{\vartheta}} ;$ therefore, $\lambda(1,1)$ $=0, \lambda(1,5)=\sqrt{4 / 5}$, and $\lambda(5,5)=(\sqrt{8} / 5)$. Thus, by Lemma 2 and after simplification,

$$
\mathfrak{R A B C}\left(G_{p, q}^{3}\right)=\left(\frac{12 \sqrt{5}}{5}+\frac{6 \sqrt{2}}{5}\right) p q-\frac{8 \sqrt{5}}{5}+\frac{2(4 p+2 q+2) \sqrt{2}}{5} .
$$

For $\mathfrak{R G A}\left(G_{p, q}^{3}\right)$ which is the reverse geometric-arithmetic index of $G_{p, q}^{3}$, from equation (4), we have $\lambda\left(\mathfrak{R}_{\theta}, \mathfrak{R}_{\vartheta}\right)=\left(2 \sqrt{\mathfrak{R}_{\theta} \times \mathfrak{R}_{\vartheta}} /\left(\mathfrak{R}_{\theta}+\mathfrak{R}_{\vartheta}\right)\right) ;$ therefore, $\lambda(1,1)=1, \lambda(1,5)=(\sqrt{5} / 3)$, and $\lambda(5,5)=1$. Thus, by Lemma 2 and after simplification,

$$
\Re \mathrm{RA}\left(G_{p, q}^{3}\right)=(6+2 \sqrt{5}) p q+4-\frac{4 \sqrt{5}}{3} .
$$

For $\mathfrak{R} \mathbb{M}_{1}\left(G_{p, q}^{3}\right)$ which is the first reverse Zagreb index of $G_{p, q}^{3}$, from equation (5), we have $\lambda\left(\mathfrak{R}_{\theta}, \mathfrak{R}_{\vartheta}\right)=\left(\mathfrak{R}_{\theta}+\mathfrak{R}_{\vartheta}\right)$; therefore, $\lambda(1,1)=2, \lambda(1,5)=6$, and $\lambda(5,5)=10$. Thus, by Lemma 2 and after simplification, 


$$
\left.\mathfrak{R M} \mathbb{M}_{1}\left(G_{p, q}^{3}\right)\right)=72 p q+32 p+16 q .
$$

For $\mathfrak{R} \nVdash M\left(G_{p, q}^{3}\right)$ which is the first reverse hyper-Zagreb index of $G_{p, q}^{3}$, from equation (6), we have $\lambda\left(\mathfrak{R}_{\theta}, \mathfrak{R}_{\vartheta}\right)=\left(\mathfrak{R}_{\theta}+\mathfrak{R}_{\vartheta}\right)^{2} ; \quad$ therefore, $\quad \lambda(1,1)=4$, $\lambda(1,5)=36$, and $\lambda(5,5)=100$. Thus, by Lemma 2 and after simplification,

$$
\mathfrak{R} \boxplus M\left(G_{p, q}^{3}\right)=528 p q+384 p+192 q+64 .
$$

For $\mathfrak{R} \mathbb{F}\left(G_{p, q}^{3}\right)$ which is the reverse forgotten index of $G_{p, q}^{3}$ from equation (7), we have $\lambda\left(\mathfrak{R}_{\theta}, \mathfrak{R}_{\vartheta}\right)=\left(\left(\mathfrak{R}_{\theta}\right)^{2}+\left(\mathfrak{R}_{\vartheta}\right)^{2}\right) ; \quad$ therefore, $\quad \lambda(1,1)=2$, $\lambda(1,5)=26$, and $\lambda(5,5)=50$. Thus, by Lemma 2 and after simplification,

$$
\mathfrak{R} \mathbb{F}\left(G_{p, q}^{3}\right)=312 p q+192 p+96 q .
$$

Theorem 6. Let $G_{p, q}^{3}$ be a third-type $p$ th chain hex-derived networks. Then, the first reverse redefined index is

$$
\Re \mathbb{R} \mathbb{Z}_{1}\left(G_{p, q}^{3}\right)=\frac{72 p q}{5}-\frac{32 p}{5}-\frac{16 q}{5} .
$$

The second reverse redefined index is

$$
\mathfrak{R} \mathbb{R} \mathbb{Z}_{2}\left(G_{p, q}^{3}\right)=14 p q+8 p+4 q+\frac{8}{3} .
$$

The third reverse redefined index is

$$
\Re \mathbb{R}_{3}\left(G_{p, q}^{3}\right)=936 p q+992 p+496 q+384 .
$$

Proof. For $\mathfrak{R} \mathbb{R} \mathbb{Z}_{1}\left(G_{p, q}^{3}\right)$ which is the first reverse redefined index of $G_{p, q}^{3}$, from equation (8), we have $\lambda\left(\mathfrak{R}_{\theta}, \mathfrak{R}_{\vartheta}\right)=\left(\mathfrak{R}_{\theta}+\mathfrak{R}_{\vartheta}\right)^{1}\left(\mathfrak{R}_{\theta} \times \mathfrak{R}_{\vartheta}\right)^{-1} ; \quad$ therefore, $\lambda(1,1)=2, \lambda(1,5)=(6 / 5)$, and $\lambda(5,5)=(2 / 5)$. Thus, by Lemma 2 and after simplification,

$$
\Re \mathbb{R} \mathbb{Z}_{1}\left(G_{p, q}^{3}\right)=\frac{72 p q}{5}-\frac{32 p}{5}-\frac{16 q}{5} .
$$

For $\mathfrak{R} \mathbb{R} \mathbb{Z}_{2}\left(G_{p, q}^{3}\right)$ which is the second reverse redefined index of $G_{p, q}^{3}$, from equation (8), we have $\lambda\left(\mathfrak{R}_{\theta}, \mathfrak{R}_{\vartheta}\right)=\left(\mathfrak{R}_{\theta}+\mathfrak{R}_{\vartheta}\right)^{-1}\left(\mathfrak{R}_{\theta} \times \mathfrak{R}_{\vartheta}\right)^{1} ; \quad$ therefore, $\lambda(1,1)=(1 / 2), \lambda(1,5)=(5 / 6)$, and $\lambda(5,5)=(5 / 2)$. Thus, by Lemma 2 and after simplification,

$$
\mathfrak{R} \mathbb{Z}_{2}\left(G_{p, q}^{3}\right)=14 p q+8 p+4 q+\frac{8}{3} .
$$

For $\mathfrak{R} \mathbb{R} \mathbb{Z}_{3}\left(G_{p, q}^{3}\right)$ which is the third reverse redefined index of $G_{p, q}^{3}$, from equation (8), we have $\lambda\left(\mathfrak{R}_{\theta}, \mathfrak{R}_{\vartheta}\right)=\left(\mathfrak{R}_{\theta}+\mathfrak{R}_{\vartheta}\right)\left(\mathfrak{R}_{\theta} \times \mathfrak{R}_{\vartheta}\right)$; therefore, $\lambda(1,1)=2$, $\lambda(1,5)=30$, and $\lambda(5,5)=250$. Thus, by Lemma 2 and after simplification,

$$
\mathfrak{R} \mathbb{R} \mathbb{Z}_{3}\left(G_{p, q}^{3}\right)=936 p q+992 p+496 q+384
$$

3.3. Results for Case 3. We provide a formula that would be used to calculate any reverse degree topological descriptors of Case 3 for $G_{p, q}^{3}$.

Lemma 3. Let $G_{p, q}^{3}$ be a third-type $p$ th chain hex-derived networks. Then,

$$
\begin{aligned}
\mathscr{T}_{3}\left(G_{p, q}^{3}\right)= & 3 p q(\lambda(1,1)+2 \lambda(1,5)+\lambda(5,5)) \\
& +2(\lambda(5,5)-\lambda(1,1))(2 p+q) \\
& +2(\lambda(1,1)-4 \lambda(1,5)+3 \lambda(5,5)) .
\end{aligned}
$$

Proof. The graph $G_{p, q}^{3}$ contains $12 p q$ edges, and maximum degree in $G_{p, q}^{3}$ graph is 8 . There are two types of reverse degree vertices in $G_{p, q}^{3}$ that are 1 and 5. Let us partition the edges of $G_{p, q}^{3}$ according to its reverse degrees according to Case 3 as

$$
\begin{aligned}
& \mathbf{E}_{1,1}=\left\{\theta \vartheta \in E\left(G_{p, q}^{3}\right): \mathfrak{R}_{\theta}=1, \mathfrak{R}_{\vartheta}=1\right\}, \\
& \mathbf{E}_{1,5}=\left\{\theta \vartheta \in E\left(G_{p, q}^{3}\right): \mathfrak{R}_{\theta}=1, \mathfrak{R}_{\vartheta}=5\right\}, \\
& \mathbf{E}_{5,5}=\left\{\theta \vartheta \in E\left(G_{p, q}^{3}\right): \mathfrak{R}_{\theta}=5, \mathfrak{R}_{\vartheta}=5\right\} .
\end{aligned}
$$

Note that $E\left(G_{p, q}^{3}\right)=\mathbf{E}_{1,1} \cup \mathbf{E}_{1,5} \cup \mathbf{E}_{5,5} \quad$ and $\left|\mathbf{E}_{1,1}\right|=3 p q-4 p-2 q+2, \quad\left|\mathbf{E}_{1,5}\right|=6 p q-8, \quad$ and $\left|\mathbf{E}_{5,5}\right|=3 p q+4 p+2 q+6$. Hence,

$$
\begin{aligned}
\mathscr{T}_{1}\left(G_{p, q}^{3}\right)= & \sum_{\theta \vartheta \in E\left(G_{p, q}^{3}\right)} \lambda\left(\mathfrak{R}_{\theta}, \mathfrak{R}_{\vartheta}\right) \\
= & \sum_{\theta \vartheta \in \mathrm{E}_{1,1}} \lambda(1,1)+\sum_{\theta \vartheta \in \mathbf{E}_{1,5}} \lambda(1,5)+\sum_{\theta \vartheta \in \mathbf{E}_{5,5}} \lambda(5,5) \\
= & (3 p q-4 p-2 q+2) \lambda(1,1)+(6 p q-8) \lambda(1,5) \\
& +(3 p q+4 p+2 q+6) \lambda(5,5) .
\end{aligned}
$$

After simplification, we obtain

$$
\begin{aligned}
\mathscr{T}_{3}\left(G_{p, q}^{3}\right)= & 3 p q(\lambda(1,1)+2 \lambda(1,5)+\lambda(5,5)) \\
& +2(\lambda(5,5)-\lambda(1,1))(2 p+q)+2(\lambda(1,1) \\
& -4 \lambda(1,5)+3 \lambda(5,5)) .
\end{aligned}
$$

Theorem 7. The general reverse Randić index of $G_{p, q}^{3}$ is equal to 


$$
\Re \mathbb{R}_{\alpha}\left(G_{p, q}^{3}\right)= \begin{cases}108 p q+96 p+48 q+112, & \text { for } \alpha=1, \\ (18+6 \sqrt{5}) p q+16 p+8 q+32-8 \sqrt{5}, & \text { for } \alpha=\frac{1}{2}, \\ \left(\frac{18}{5}+\frac{6 \sqrt{5}}{5}\right) p q-\frac{16 p}{5}-\frac{8}{5} q+\frac{16}{5}-\frac{8 \sqrt{5}}{5}, & \text { for } \alpha=\frac{-1}{2}, \\ \frac{108 p q}{25}-\frac{96 p}{25}-\frac{48 q}{25}+\frac{16}{25}, & \text { for } \alpha=-1 .\end{cases}
$$

Proof. For $\mathfrak{R} \mathbb{R}_{\alpha}\left(G_{p, q}^{3}\right)$ which is the general reverse Randić index of $G_{p, q}^{3}$, from equation (2), we have
$\lambda\left(\mathfrak{R}_{\theta}, \mathfrak{R}_{\vartheta}\right)=\left(\mathfrak{R}_{\theta} \times \mathfrak{R}_{\vartheta}\right)^{\alpha} ; \quad$ therefore, $\quad \lambda(1,1)=1$, $\lambda(1,5)=(5)^{\alpha}$, and $\lambda(5,5)=(25)^{\alpha}$. Thus, by Lemma 3 ,

$$
\left.\mathfrak{R} \mathbb{R}_{\alpha}\left(G_{p, q}^{3}\right)\right)=3 p q\left(1+2(5)^{\alpha}+(25)^{\alpha}\right)+2\left((25)^{\alpha}-1\right)(2 p+q)+2\left(1-4(5)^{\alpha}+3(25)^{\alpha}\right) .
$$

Put $\alpha=1$, and we have

$$
\left.\mathfrak{R} \mathbb{R}_{1}\left(G_{p, q}^{3}\right)\right)=108 p q+96 p+48 q+112 .
$$

Put $\alpha=(1 / 2)$, and we have

$$
\left.\mathfrak{R} \mathbb{R}_{(1 / 2)}\left(G_{p, q}^{3}\right)\right)=(18+6 \sqrt{5}) p q+16 p+8 q+32-8 \sqrt{5} \text {. }
$$

Put $\alpha=(-1 / 2)$, and we have

$$
\left.\mathfrak{R} \mathbb{R}_{(-1 / 2)}\left(G_{p, q}^{3}\right)\right)=\left(\frac{18}{5}+\frac{6 \sqrt{5}}{5}\right) p q-\frac{16 p}{5}-\frac{8}{5} q+\frac{16}{5}-\frac{8 \sqrt{5}}{5} .
$$

Put $\alpha=-1$, and we have

$$
\left.\Re \mathbb{R}_{-1}\left(G_{p, q}^{3}\right)\right)=\frac{108 p q}{25}-\frac{96 p}{25}-\frac{48 q}{25}+\frac{16}{25} .
$$

Theorem 8. Let $G_{p, q}^{3}$ be a third-type $p$ th chain hex-derived networks. Then, the reverse atom-bond connectivity index is

$$
\mathfrak{R A B C}\left(G_{p, q}^{3}\right)=\left(\frac{12 \sqrt{5}}{5}+\frac{6 \sqrt{2}}{5}\right) p q-\frac{16 \sqrt{5}}{5}+\frac{2(4 p+2 q+6) \sqrt{2}}{5} .
$$

The reverse geometric-arithmetic index is

$$
\mathfrak{R G A}\left(G_{p, q}^{3}\right)=(6+2 \sqrt{5}) p q+8-\frac{8 \sqrt{5}}{3} .
$$

The first reverse Zagreb index is

$$
\left.\mathfrak{R M} \mathbb{M}_{1}\left(G_{p, q}^{3}\right)\right)=72 p q+32 p+16 q+16 .
$$

The reverse hyper-Zagreb index is

$$
\mathfrak{R} \nVdash M\left(G_{p, q}^{3}\right)=528 p q+384 p+192 q+320 .
$$

The reverse forgotten index is

$$
\mathfrak{R} \mathbb{F}\left(G_{p, q}^{3}\right)=312 p q+192 p+96 q+96 .
$$

Proof. For $\mathfrak{R A B C}\left(G_{p, q}^{3}\right)$ which is the reverse atom-bond connectivity index of $G_{p, q}^{3}$, from equation (3), we have
$\lambda\left(\mathfrak{R}_{\theta}, \mathfrak{R}_{\vartheta}\right)=\sqrt{\mathfrak{R}_{\theta}+\mathfrak{R}_{\vartheta}-2 / \mathfrak{R}_{\theta} \times \mathfrak{R}_{9}} ;$ therefore, $\lambda(1,1)=0$, $\lambda(1,5)=\sqrt{4 / 5}$, and $\lambda(5,5)=(\sqrt{8} / 5)$. Thus, by Lemma 3 and after simplification,

$$
\Re \mathbb{A} \mathbb{B C}\left(G_{p, q}^{3}\right)=\left(\frac{12 \sqrt{5}}{5}+\frac{6 \sqrt{2}}{5}\right) p q-\frac{16 \sqrt{5}}{5}+\frac{2(4 p+2 q+6) \sqrt{2}}{5} .
$$

For $\Re G A\left(G_{p, q}^{3}\right)$ which is the reverse geometric-arithmetic index of $G_{p, q}^{3}$, from equation (4), we have $\lambda\left(\mathfrak{R}_{\theta}, \mathfrak{R}_{\vartheta}\right)=\left(2 \sqrt{\mathfrak{R}_{\theta} \times \mathfrak{R}_{\vartheta}} / \mathfrak{R}_{\theta}+\mathfrak{R}_{\vartheta}\right)$; therefore, $\lambda(1,1)=1$, $\lambda(1,5)=(\sqrt{5} / 3)$, and $\lambda(5,5)=1$. Thus, by Lemma 3 and after simplification,

$$
\mathfrak{R G A}\left(G_{p, q}^{3}\right)=(6+2 \sqrt{5}) p q+8-\frac{8 \sqrt{5}}{3} .
$$


TABLE 1: Numerical comparison of $\mathfrak{R A B C}, \mathfrak{R G A}, \mathfrak{R} \mathbb{M}_{1}, \mathfrak{R} \llbracket \mathrm{M}$, and $\mathfrak{R} \mathbb{F}$ for Case 1 .

\begin{tabular}{|c|c|c|c|c|c|}
\hline$[p, q]$ & $\Re \mathbb{A} B \mathbb{C}$ & $\Re$ GA & $\mathfrak{R} \mathbb{M}_{1}$ & $\mathfrak{R} \Vdash M$ & $\mathfrak{R} \mathbb{F}$ \\
\hline$[1,1]$ & 8.0114 & 11.491 & 120 & 1168 & 600 \\
\hline$[2,2]$ & 32.597 & 42.907 & 384 & 3328 & 1824 \\
\hline$[3,3]$ & 71.309 & 95.269 & 792 & 6544 & 3672 \\
\hline$[4,4]$ & 124.15 & 168.57 & 1344 & 10816 & 6144 \\
\hline$[5,5]$ & 191.12 & 262.82 & 2040 & 16144 & 9240 \\
\hline$[6,6]$ & 272.21 & 378.02 & 2880 & 22528 & 12960 \\
\hline$[7,7]$ & 367.43 & 514.16 & 3864 & 29968 & 17304 \\
\hline$[8,8]$ & 476.78 & 671.25 & 4992 & 38464 & 22272 \\
\hline$[9,9]$ & 600.26 & 849.27 & 6264 & 48016 & 27864 \\
\hline$[10,10]$ & 737.87 & 1048.2 & 7680 & 58624 & 34080 \\
\hline
\end{tabular}

TABLE 2: Numerical comparison of $\mathfrak{R A B C}, \mathfrak{R G A}, \mathfrak{R M}, \mathfrak{R} \Vdash \mathrm{M}$, and $\mathfrak{R} \mathbb{F}$ for Case 2.

\begin{tabular}{|c|c|c|c|c|c|}
\hline$[\mathbf{p}, \mathbf{q}]$ & $\Re A \mathbb{B C}$ & $\Re \mathrm{GA}$ & $\mathfrak{R M} \mathbb{M}_{1}$ & $\mathfrak{R} \nVdash M$ & $\mathfrak{R F}$ \\
\hline$[5,6]$ & 227.56 & 315.19 & 2416 & 18976 & 10896 \\
\hline$[5,7]$ & 264.01 & 367.55 & 2792 & 21808 & 12552 \\
\hline$[5,8]$ & 300.47 & 419.91 & 3168 & 24640 & 14208 \\
\hline$[4,4]$ & 124.15 & 168.57 & 1344 & 10816 & 6144 \\
\hline$[6,6]$ & 272.21 & 378.02 & 2880 & 22528 & 12960 \\
\hline$[8,8]$ & 476.78 & 671.25 & 4992 & 38464 & 22272 \\
\hline$[9,8]$ & 535.55 & 755.02 & 5600 & 43072 & 24960 \\
\hline$[9,7]$ & 470.85 & 660.77 & 4936 & 38128 & 22056 \\
\hline$[10,8]$ & 594.32 & 838.80 & 6208 & 47680 & 27648 \\
\hline$[12,10]$ & 883.66 & 1257.7 & 9184 & 69952 & 40704 \\
\hline
\end{tabular}

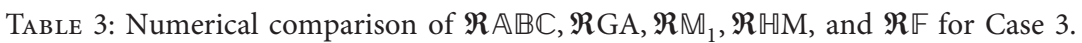

\begin{tabular}{|c|c|c|c|c|c|}
\hline$[\mathbf{p}, \mathbf{q}]$ & $\mathfrak{R A B C}$ & $\Re \mathrm{GA}$ & $\mathfrak{R M} \mathbb{M}_{1}$ & $\mathfrak{R} \backsim M$ & $\mathfrak{R} \mathbb{F}$ \\
\hline$[4,5]$ & 152.22 & 211.48 & 1664 & 13376 & 7584 \\
\hline$[6,7]$ & 314.41 & 441.87 & 3344 & 26144 & 15024 \\
\hline$[8,9]$ & 533.10 & 756.03 & 5600 & 43136 & 24960 \\
\hline$[10,11]$ & 808.31 & 1154.0 & 8432 & 64352 & 37392 \\
\hline$[12,13]$ & 1140.0 & 1635.7 & 11840 & 89792 & 52320 \\
\hline$[4,3]$ & 93.448 & 127.70 & 1056 & 8768 & 4896 \\
\hline$[6,5]$ & 227.38 & 316.20 & 2448 & 19424 & 11088 \\
\hline$[8,7]$ & 417.83 & 588.47 & 4416 & 34304 & 19776 \\
\hline$[10,9]$ & 664.78 & 944.53 & 6960 & 53408 & 30960 \\
\hline$[12,11]$ & 968.24 & 1384.4 & 10080 & 76736 & 44640 \\
\hline
\end{tabular}

For $\mathfrak{R} \mathbb{M}_{1}\left(G_{p, q}^{3}\right)$ which is the first reverse Zagreb index of $G_{p, q}^{3}$, from equation (5), we have $\lambda\left(\mathfrak{R}_{\theta}, \mathfrak{R}_{\vartheta}\right)=\left(\mathfrak{R}_{\theta}+\mathfrak{R}_{\vartheta}\right)$; therefore, $\lambda(1,1)=2, \lambda(1,5)=6$, and $\lambda(5,5)=10$. Thus, by Lemma 3 and after simplification,

$$
\left.\mathfrak{R M} \mathbb{M}_{1}\left(G_{p, q}^{3}\right)\right)=72 p q+32 p+16 q+16 .
$$

For $\mathfrak{R} \backsim M\left(G_{p, q}^{3}\right)$ which is the first reverse hyper-Zagreb index of $G_{p, q}^{3}$, from equation (6), we have $\lambda\left(\mathfrak{R}_{\theta}, \mathfrak{R}_{\vartheta}\right)=\left(\mathfrak{R}_{\theta}+\mathfrak{R}_{\vartheta}\right)^{2} ; \quad$ therefore, $\quad \lambda(1,1)=4$, $\lambda(1,5)=36$, and $\lambda(5,5)=100$. Thus, by Lemma 3 and after simplification,

$$
\mathfrak{R} \nVdash \mathrm{M}\left(G_{p, q}^{3}\right)=528 p q+384 p+192 q+320 .
$$

For $\mathfrak{R} \mathbb{F}\left(G_{p, q}^{3}\right)$ which is the reverse forgotten index of $G_{p, q}^{3}$, from equation (7), we have $\lambda\left(\mathfrak{R}_{\theta}, \mathfrak{R}_{\vartheta}\right)$
$=\left(\left(\mathfrak{R}_{\theta}\right)^{2}+\left(\mathfrak{R}_{\vartheta}\right)^{2}\right)$; therefore, $\lambda(1,1)=2, \lambda(1,5)=26$ and $\lambda(5,5)=50$. Thus, by Lemma 3 and after simplification,

$$
\mathfrak{R} \mathbb{F}\left(G_{p, q}^{3}\right)=312 p q+192 p+96 q+96 .
$$

Theorem 9. Let $G_{p, q}^{3}$ be a third-type $p$ th chain hex-derived networks. Then, the first reverse redefined index is

$$
\Re \mathbb{R} \mathbb{Z}_{1}\left(G_{p, q}^{3}\right)=\frac{72 p q}{5}-\frac{32 p}{5}-\frac{16 q}{5}-\frac{16}{5} .
$$

The second reverse redefined index is

$$
\mathfrak{R} \mathbb{Z}_{2}\left(G_{p, q}^{3}\right)=14 p q+8 p+4 q+\frac{28}{3} .
$$

The third reverse redefined index is 


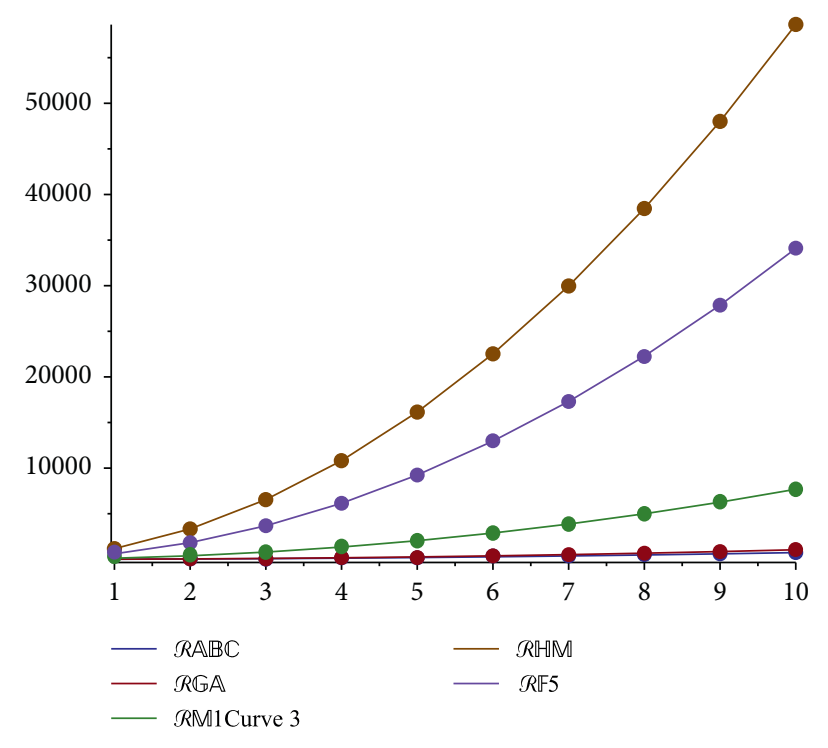

Figure 4: Graphical representation of Table 1.

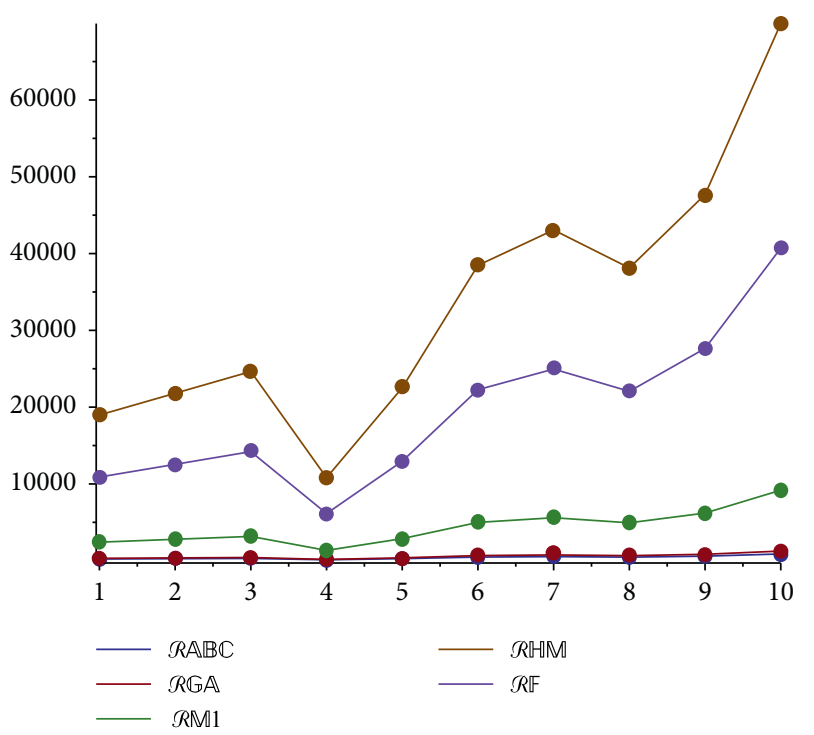

Figure 5: Graphical representation of Table 2.

$$
\mathfrak{R} \mathbb{R} \mathbb{Z}_{3}\left(G_{p, q}^{3}\right)=936 p q+992 p+496 q+1264
$$

Proof. For $\mathfrak{R} \mathbb{R} \mathbb{Z}_{1}\left(G_{p, q}^{3}\right)$ which is the first reverse redefined index of $G_{p, q}^{3}$, from equation (8), we have $\lambda\left(\mathfrak{R}_{\theta}, \mathfrak{R}_{\vartheta}\right)=\left(\mathfrak{R}_{\theta}+\mathfrak{R}_{\vartheta}\right)^{1}\left(\mathfrak{R}_{\theta} \times \mathfrak{R}_{\vartheta}\right)^{-1} ;$ therefore, $\lambda(1,1)$ $=2, \lambda(1,5)=(6 / 5)$, and $\lambda(5,5)=(2 / 5)$. Thus, by Lemma 3 and after simplification,

$$
\mathfrak{R} \mathbb{R} \mathbb{Z}_{1}\left(G_{p, q}^{3}\right)=\frac{72 p q}{5}-\frac{32 p}{5}-\frac{16 q}{5}-\frac{16}{5}
$$

For $\mathfrak{R} \mathbb{R} \mathbb{Z}_{2}\left(G_{p, q}^{3}\right)$ which is the second reverse redefined index of $G_{p, q}^{3}$, from equation (8), we have
$\lambda\left(\mathfrak{R}_{\theta}, \mathfrak{R}_{\vartheta}\right)=\left(\mathfrak{R}_{\theta}+\mathfrak{R}_{\vartheta}\right)^{-1}\left(\mathfrak{R}_{\theta} \times \mathfrak{R}_{\vartheta}\right)^{1} ;$ therefore, $\lambda(1,1)$ $=(1 / 2), \lambda(1,5)=(5 / 6)$, and $\lambda(5,5)=(5 / 2)$. Thus, by Lemma 3 and after simplification,

$$
\mathfrak{R} \mathbb{Z}_{2}\left(G_{p, q}^{3}\right)=14 p q+8 p+4 q+\frac{28}{3} .
$$

For $\Re \mathbb{R} \mathbb{Z}_{3}\left(G_{p, q}^{3}\right)$ which is the third reverse redefined index of $G_{p, q}^{3}$, from equation (8), we have $\lambda\left(\mathfrak{R}_{\theta}, \mathfrak{R}_{\vartheta}\right)=\left(\mathfrak{R}_{\theta}+\mathfrak{R}_{\vartheta}\right)\left(\mathfrak{R}_{\theta} \times \mathfrak{R}_{\vartheta}\right)$; therefore, $\lambda(1,1)=2$, $\lambda(1,5)=30$, and $\lambda(5,5)=250$. Thus, by Lemma 3 and after simplification,

$$
\Re \mathbb{R} \mathbb{Z}_{3}\left(G_{p, q}^{3}\right)=936 p q+992 p+496 q+1264
$$




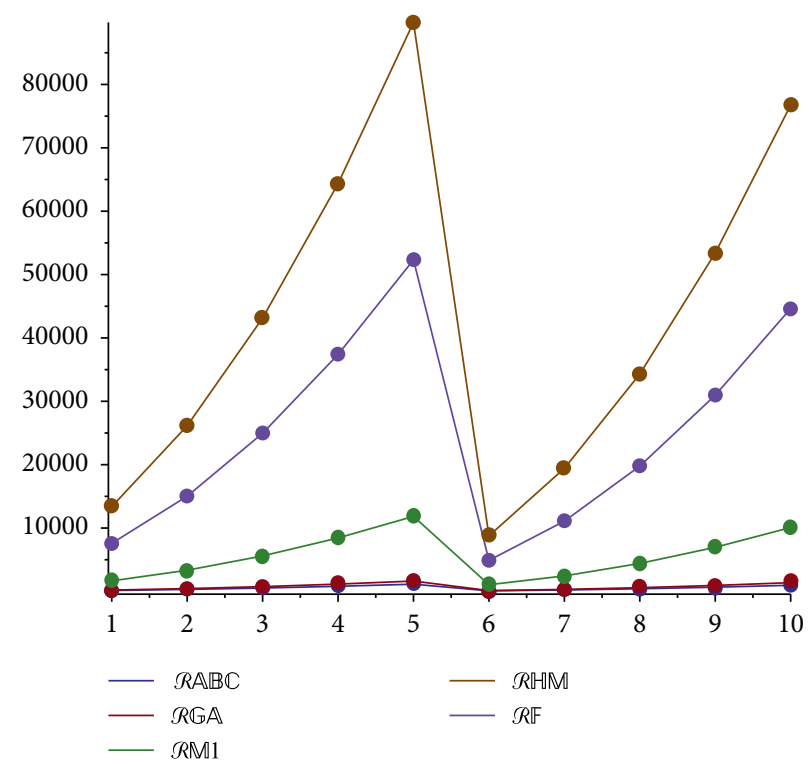

Figure 6: Graphical representation of Table 3.

\section{Numerical and Graphical Representation}

In this section, we determine the numerical values of $\mathfrak{R A B C}, \mathfrak{R G A}, \mathfrak{R M}, 1, \mathfrak{R} \backsim M$, and $\mathfrak{R} \mathbb{F}$ in Tables $1-3$, for Case 1 , Case 2, and Case 3, respectively. We represent these results graphically in Figures 4-6.

\section{Data Availability}

No data were used to support the findings of the study.

\section{Conflicts of Interest}

The authors declare that they have no conflicts of interest.

\section{References}

[1] F. Simonraj and A. George, "On the metric dimension of $\mathrm{HDN}_{3}$ and $\mathrm{PHDN}_{3}$," in Proceedings of the IEEE International Conference on Power, Control, Signals and Instrumentation Engineering (ICPCSI), pp. 1333-1336, Chennai, India, September 2017.

[2] H. Ali, M. A. Binyamin, M. K. Shafiq, and W. Gao, "On the degree-based topological indices of some derived networks," Mathematics, vol. 7, no. 7, p. 612, 2019.

[3] C.-C. Wei, H. Ali, M. A. Binyamin, M. N. Naeem, and J.-B. Liu, "Computing degree based topological properties of third type of hex-derived networks," Mathematics, vol. 7, no. 4 , p. $368,2019$.

[4] M. Karelson, Molecular Descriptors in QSAR/QSPR, Wiley, New York, NY, USA, 2000.

[5] A. Aslam, Y. Bashir, S. Ahmad, and W. Gao, "On topological indices of certain dendrimer structures," Zeitschrift für Naturforschung, vol. 72, no. 6, pp. 559-566, 2017.

[6] M. Bača, J. Horváthová, M. Mokrišová, and A. Suhányiová, "On topological indices of fullerenes," Applied Mathematics and Computation, vol. 251, pp. 154-161, 2015.

[7] A. Q. Baig, M. Imran, and H. Ali, "On topological indices of poly oxide, poly silicate, DOX, and DSL networks," Canadian Journal of Chemistry, vol. 93, no. 7, pp. 730-739, 2015.
[8] S. Hayat and M. Imran, "Computation of topological indices of certain networks," Applied Mathematics and Computation, vol. 240, pp. 213-228, 2014.

[9] A. Koam and A. Ahmad, "Polynomials of degree-based indices for three-dimensional mesh network," Computers, Materials \& Continua, vol. 65, no. 2, pp. 1271-1282, 2020.

[10] C. Rui, G. Ali, G. Rahmat, M. Y. Khan, A. SemanicovaFenovcikova, and J.-B. Liu, "Investigation of general power sum-connectivity index for some classes of extremal graphs," Complexity, vol. 2021, Article ID 6623277, 6 pages, 2021.

[11] G. Hong, Z. Gu, M. Javaid, H. M. Awais, and M. K. Siddiqui, "Degree-based topological invariants of metal-organic networks," IEEE Access, vol. 8, pp. 68288-68300, 2020.

[12] Z. Iqbal, A. Aslam, M. Ishaq, and M. Aamir, "Characteristic study of irregularity measures of some nanotubes," Canadian Journal of Physics, vol. 97, no. 10, pp. 1125-1132, 2019.

[13] V. R. Kulli, "Reverse Zagreb and reverse hyper-Zagreb indices and their polynomials of rhombus silicate networks," Annals of Pure and Applied Mathematics, vol. 16, no. 1, pp. 47-51, 2018.

[14] A. Ahmad, "On the degree based topological indices of benzene ring embedded in P-type-surface in 2D network," Hacettepe Journal of Mathematics and Statistics, vol. 47, no. 1, pp. 9-18, 2018.

[15] M. Imran, A. Q. Baig, and H. Ali, "On molecular topological properties of hex-derived networks," Journal of Chemometrics, vol. 30, no. 3, pp. 121-129, 2016.

[16] D. Zhao, Y.-M. Chu, M. K. Siddiqui et al., "On reverse degree based topological indices of polycyclic metal organic network," Polycyclic Aromatic Compounds, vol. 5, 2021.

[17] A. Ahmad, "Comparative study of ve-degree and ev-degree topological descriptors for benzene ring embedded in p-typesurface in 2d network," Polycyclic Aromatic Compounds, vol. 5, 2020.

[18] J. Zhang, M. K. Siddiqui, A. Rauf, and M. Ishtiaq, "On vedegree and ev-degree based topological properties of single walled titanium dioxide nanotube," Journal of Cluster Science, 2020.

[19] N. Zahra, M. Ibrahim, and M. K. Siddiqui, "On topological indices for swapped networks modeled by optical transpose 
interconnection system," IEEE Access, vol. 8, pp. 200091200099, 2020.

[20] Z. Raza, "Leap Zagreb connection numbers for some networks models," Indonesian Journal of Chemistry, vol. 20, no. 6, pp. 1407-1413, 2020.

[21] M. K. Siddiqui, M. Imran, and A. Ahmad, "On Zagreb indices, Zagreb polynomials of some nanostar dendrimers," Applied Mathematics and Computation, vol. 280, pp. 132-139, 2016.

[22] A. Aslam, S. Ahmad, and W. Gao, "On certain topological indices of boron triangular nanotubes," Zeitschrift für Naturforschung A, vol. 72, no. 8, pp. 711-716, 2017.

[23] M. Javaid, H. Zafar, Q. Zhu, and A. M. Alanazi, "Improved lower bound of LFMD with applications of prism-related networks," Mathematical Problems in Engineering, vol. 2021, Article ID 9950310, 9 pages, 2021.

[24] J.-B. Liu, Z. Raza, and M. Javaid, "Zagreb connection numbers for cellular neural networks," Discrete Dynamics in Nature and Society, vol. 2020, Article ID 8038304, 8 pages, 2020.

[25] E. A. Refaee and A. Ahmad, "A study of hexagon star network with vertex-edge-based topological descriptors," Complexity, vol. 2021, Article ID 9911308, 7 pages, 2021.

[26] M.-S. Chen, K. G. Shin, and D. D. Kandlur, "Addressing, routing, and broadcasting in hexagonal mesh multiprocessors," IEEE Transactions on Computers, vol. 39, no. 1, pp. $10-18,1990$.

[27] A. N. A. Koam, A. Ahmad, A. Ahmad, and Y. Ahmad, "Computation of reverse degree-based topological indices of hex-derived networks," AIMS Mathematics, vol. 6, no. 10, pp. 11330-11345, 2021.

[28] Y. Huo, H. Ali, M. A. Binyamin, S. S. Asghar, U. Babar, and J.-B. Liu, "On topological indices of mth chain hex-derived network of third type," Frontiers in Physics, vol. 8, Article ID 593275, 2020.

[29] A. N. A. Koam, A. Ahmad, and M. F. Nadeem, "Comparative study of valency-based topological descriptor for hexagon star network," Computer Systems Science and Engineering, vol. 36, no. 2, pp. 293-306, 2021.

[30] H. Ali, A. Sajjad, and A. Sajjad, "On further results of hex derived networks," Open Journal of Discrete Applied Mathematics, vol. 2, no. 1, pp. 32-40, 2019.

[31] M. Imran, A. Q. Baig, S. U. Rehman, H. Ali, and R. Hasni, "Computing topological polynomials of mesh-derived networks," Discrete Mathematics, Algorithms and Applications, vol. 10, no. 6, Article ID 1850077, 2018.

[32] M. Imran, A. Q. Baig, and H. Ali, "On topological properties of dominating David derived networks," Canadian Journal of Chemistry, vol. 94, pp. 137-148, 2015.

[33] A. Aslam, S. Ahmad, and W. Gao, "On certain topological indices of boron triangular nanotubes," Zeitschrift für Naturforschung, vol. 72, no. 8, pp. 711-716, 2017. 